\title{
Effect of Cyclic Thermal Loadings on the Microstructural Evolution of a Cantor Alloy in 3D Printing Processes
}

\author{
H Wang ${ }^{1 *}$, ZG Zhu ${ }^{2}$, HS Chen ${ }^{1,3}$, SM. Nai ${ }^{2}$, XH An ${ }^{1}$, RK Zheng ${ }^{3}$, S Primig ${ }^{4}$, SP Ringer ${ }^{1}$ and XZ Liao ${ }^{1}$ \\ 1. School of Aerospace, Mechanical and Mechatronic Engineering, The University of Sydney, Sydney, \\ NSW 2006, Australia. \\ 2. Singapore Institute of Manufacturing Technology, 73 Nanyang Drive, 637662, Singapore. \\ 3. School of Physics, The University of Sydney, NSW 2006, Australia. \\ 4. School of Materials Science \& Engineering, UNSW, Sydney, NSW 2052, Australia. \\ * Corresponding author: hwan4809@uni.sydney.edu.au
}

$3 \mathrm{D}$ printing or additive manufacturing has attracted significant research attention because it is the only technique for direct fabrication of parts with complex geometries. The 3D printing processes of metallic materials consist of cyclic rapid thermal loadings that affect significantly the microstructure and consequently the mechanical properties of materials. Yet it has not been clear how the thermal history (number of cyclic thermal loadings) during the 3D printing processes affects the microstructure of materials.

Here we used a FeCoCrMnNi high-entropy alloy, known as a Cantor alloy, as the model material to explore the effect of the number of cyclic thermal loadings on the microstructure of 3D printed materials. Selective laser melting was used to print a sample. The cubic sample with a dimension of $10 \times 10 \times 10$ $\mathrm{mm}^{3}$ sample was fabricated layer-by-layer with the layer thickness of $40 \mu \mathrm{m}$. Transmission electron microscopy (TEM) was used to study nano-scale local structural features in different layers. Atom probe tomography (APT) was used to characterize the evolution of elemental distribution.

Results showed that with the increase of the number of thermal loadings, the microstructure evolved from a nanocrystalline structure (Figs. 1a), through coarse grains with cellular dislocation structures (Fig. 1b), to nanotwin (Fig. 1c) and hierarchical nanotwin structures (Fig. 1d). The evolution of the microstructure impacts significantly on the elemental distribution. An example is shown in Fig. 1e in which significant $\mathrm{Mn}$ and Ni enrichment at a dislocation cell wall area was observed. The impact of local microstructure and elemental distribution on the mechanical properties and thermal stability of the material will be discussed in detail. 

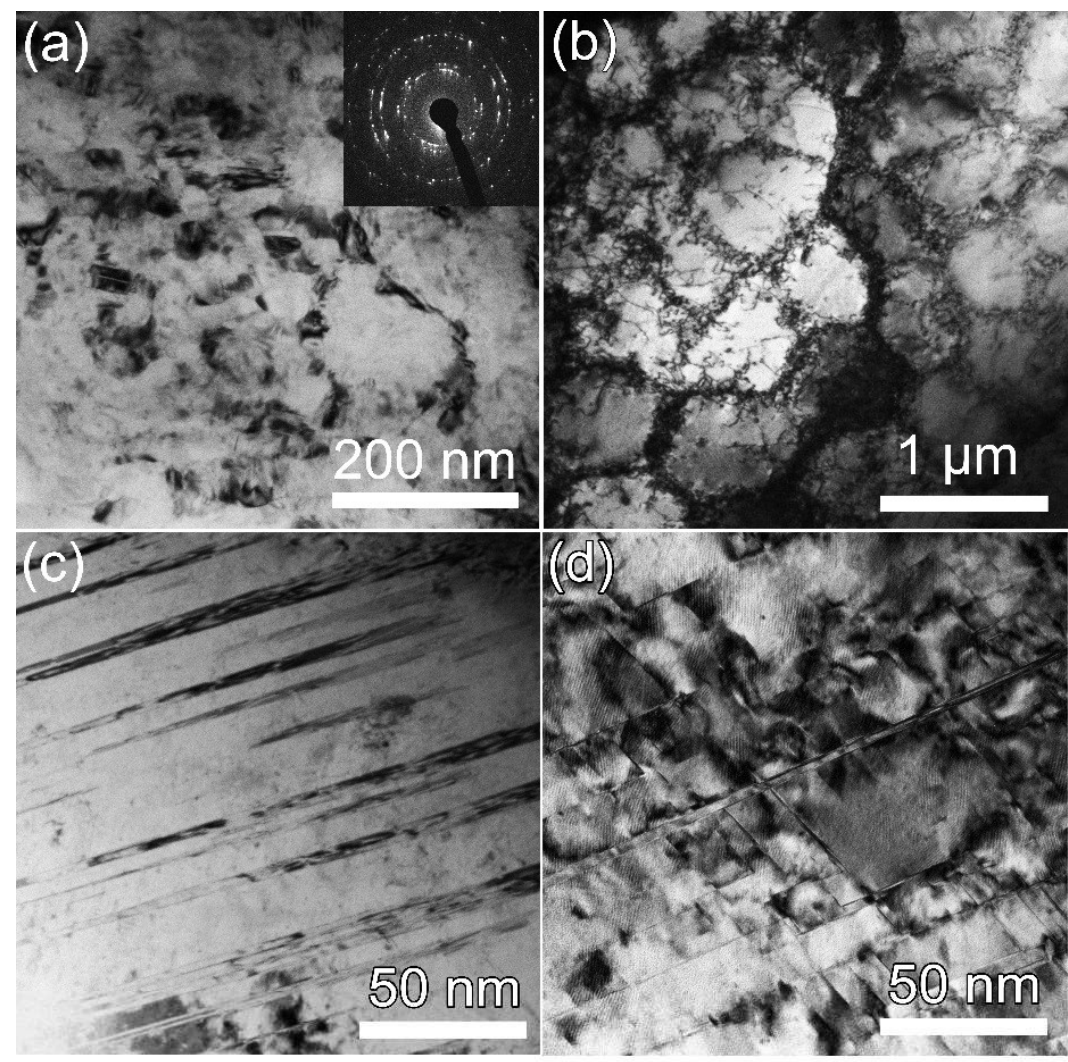

(e)
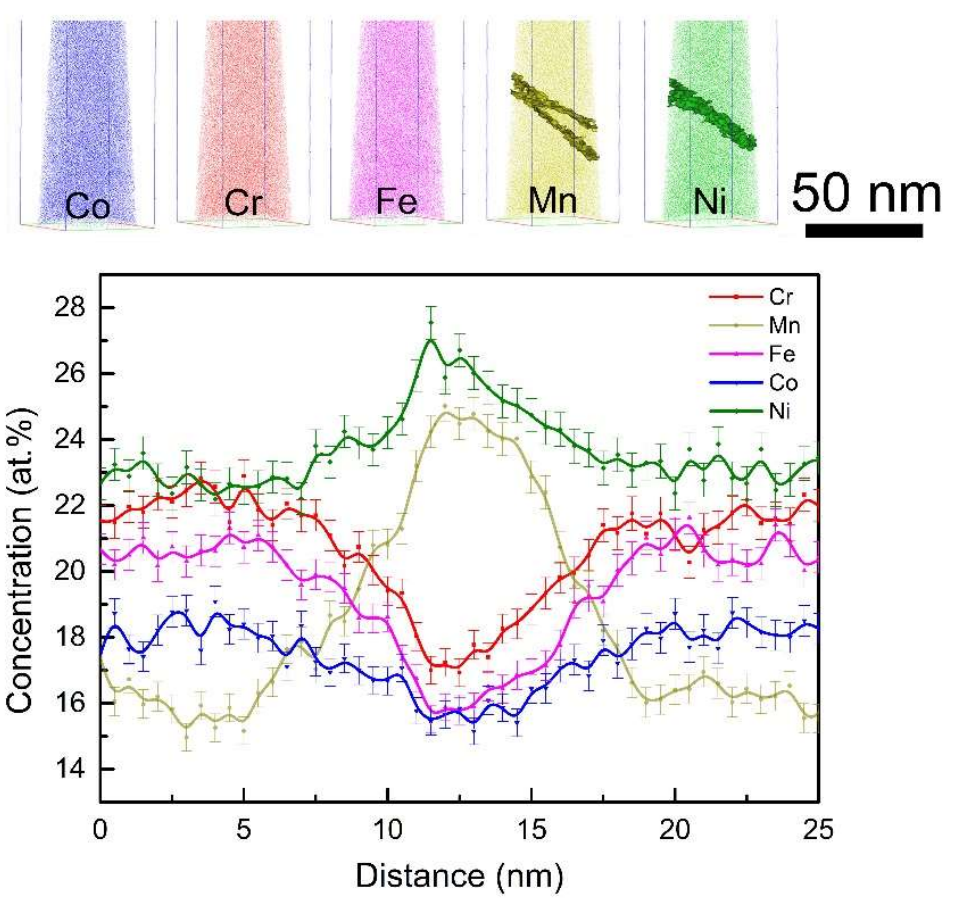

Figure 1. Typical TEM images of (a) the nanocrystalline structure in the top layer in which the number of thermal loading was 1 ; (b) an equiaxed cellular dislocation structure in coarse grains in a middle layer with the number of cyclic thermal loadings of $\sim 125$; (c and d) nanotwin and secondary nanotwin structures in the bottom layer with the number of cyclic thermal loadings of $\sim 250$. Inset on the upper right corner of (a) is a corresponding electron diffraction pattern. (e) APT data from a middle layer, indicating Mn and Ni segregation to a dislocation cell wall. 